\title{
Cytodiagnosis of histoid leprosy
}

\author{
A. S. BHAKE*, K. V. DESIKAN** \& U. N. JAJOO* \\ *Mahatma Gandhi Institute of Medical Sciences and **Leprosy \\ Histopathology Centre (LEPRA India), SEVAGRAM-442 102, India
}

\author{
Accepted for publication 17 January 2001
}

\begin{abstract}
Summary This paper presents cytomorphological features of the histoid variety of lepromatous leprosy. Fine needle aspiration of a lepromatous nodule showed cytological features consistent with those of histoid leprosy. Simultaneously, a biopsy of the nodule was also performed and the case confirmed as histoid leprosy. The advantages of the fine needle aspiration technique are that it is simple, quickly reportable, and less traumatizing. Multiple aspirations from different sites may be obtained, which would add to the value of sampling. The need to differentiate a histoid nodule from a conventional lepromatous nodule is explained.
\end{abstract}

\section{Introduction}

Reports on primary cytodiagnosis of leprosy lesions by fine needle aspiration cytology (FNAC) from skin, nerve and lymph nodes are very few in the literature. A few reports of lepromatous tuberculoid leprosy and reactions along with cytomorphological features have appeared in recent literature. ${ }^{1-4}$ However, to date, cytodiagnosis of histoid leprosy on FNAC has not been described. The present report describes for the first time the cytomorphological diagnostic features of histoid leprosy by FNAC, confirmed by biopsy.

\section{Materials and methods}

A 28 year old man presented himself at the outpatient department with the complaint of nodular swelling on the upper limbs and trunk, which had appeared 3 months earlier. On examination, several nodules $1-1.5 \mathrm{~cm}$ diameter were seen on the upper limbs, legs and dorsum of the feet. One nodule on the foot was ulcerated. A nodule on the right forearm and one on the left foot were seen on thickened palpable cutaneous nerves, Skin on back, arms and thighs was oily and shining. There was infiltration on the earlobes. The nodules had a punched-out appearance and were firm. Skin smears were highly positive for AFB, with a $\mathrm{BI}$ of $5+$, the smears from two nodules being $6+$ on the Ridley scale. The clinical diagnosis was of lepromatous leprosy with nodules. A nodule on the right side of neck was selected for FNAC. 
The nodule selected for FNAC measured $1 \mathrm{~cm} \times 1 \mathrm{~cm}$ and was raised relative to the skin surface. It was situated on the right side of the neck in the mid-lateral position. There was no other swelling or enlarged lymph nodes in the neck. Haematological investigations were within normal limits. There was no fever associated with the nodule.

FNAC was carried out by conventional techniques using a $24 \mathrm{sw}$ disposable needle. The aspirate was thick, white and soft with negligible contamination with blood. A second aspiration was done, with similar results. Four smears were made from each of the aspirates. From each set, two smears were fixed in 95\% ethyl alcohol for Papanicolaou staining; one was air dried and stained by Giemsa stain, the other was also air dried and stained by modified Zeihl-Neelson stain.

\section{Findings}

The smears showed adequate cellularity. The architectural pattern observed was of two types in the two sets of smears. The first pattern seen in one set of smears was of multilayered cell pallisades of spindle-shaped histocytes on the endothelial vascular core (Figures 1 and 2). Characteristic cells of this pattern showed spindle-shaped cells with round and elliptical blunt nuclei having fine regular chromatin and small nucleoli. There was vesiculation in a few nuclei. Intercellular stroma between these cells was nearly absent.

The second pattern seen in the other set of smears was of isolated cells of polygonal shape with ample cytoplasm (Figure 3). The cells were isolated and in loose groups. These polygonal cell showed abundant cytoplasm which was multivacuolated, presenting a soap bubble appearance. The nuclei in these cells were centrally or eccentrically located. They showed fine chromation with a visible nucleolus. At places there were multinucleated giant cells with the same cytoplasmic character as that of single vacuolated cells. Another characteric feature which was observed in some of the cells was of negative image (intracytoplasmic unstained spaces) seen as small crystalloid spaces in the Giemsa-stained material. The background showed fine and coarse vacuolated material that appeared to be cytoplasmic sheddings. The background also contained negligible lymphocytes.

The smears of both sets stained by the modified Zeihl-Neelsen method showed Intracytoplasmic bundles of acid-fast bacilli.

Histological examination showed a circumscribed granuloma in the dermis, separated from the epidermis by a narrow, clear sub-epidermal zone. The granuloma consisted of thin spindle-shaped cells forming interlacing bands whorls and tight curlicues (Figure 4). Fite's stain showed the cells containing packed acid-fast bacilli with characteristic 'histoid habitus'. Deeper down in the dermis, a classical lepromatous granuloma was seen, composed of sheets of vacuolated macrophages (lepra cells) with moderate infiltration by plasma cells and lymphocytes. These features confirm that this was a case of histoid leprosy and the FNAC findings reflected the histological findings.

\section{Discussion}

Cytodiagnosis by FNAC has certain advantages over reporting by excision biopsy. The technique is simple and is less traumatic. As such, it is possible to obtain tissue material from multiple sites and from lesions in different locations that would add to the value of 


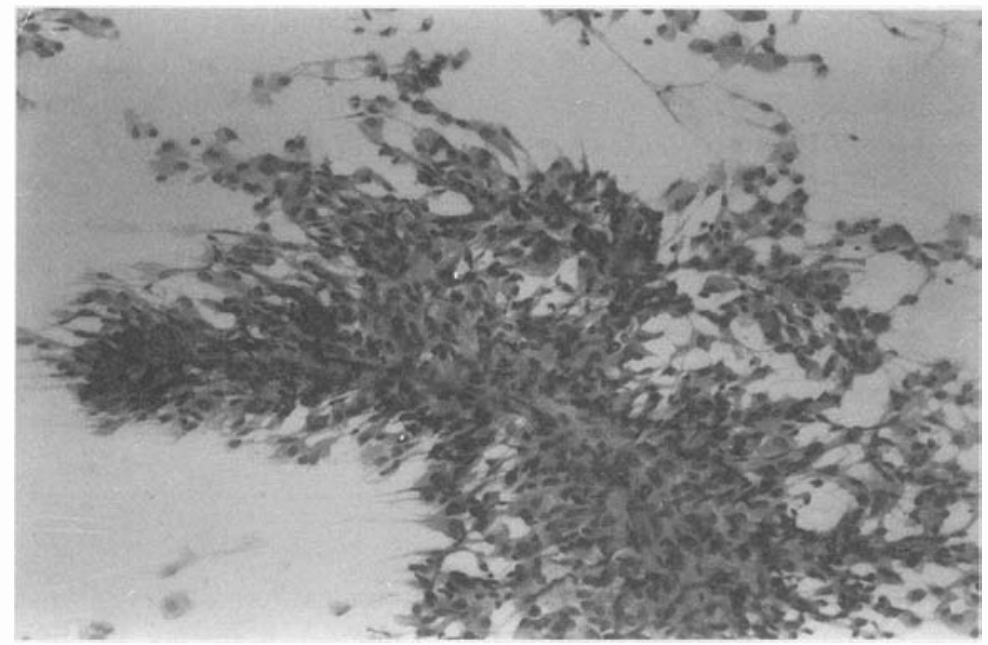

Figure 1. Photomicrograph of FNAC preparation showing multilayered palisades of spindle shaped histocytes. $($ Papanicolaou $\times 100)$.

sampling. Processing the smears, staining and reporting can be done immediately, so that the report is available to the treating physician while the patient waits in the out-patient clinic. Morphologically, the cells could be visualized in more or less their normal shape, since they undergo less shrinkage and distortion. However, FNAC cannot replace excision biopsy where the architecture of the lesion and its relation to other structures can be visualized. Further, by excision biopsy, a larger size of tissue is obtained so that multiple and deeper sections can be obtained.

In the present paper, the cell morphology is clear and evident. The arrangement of cells

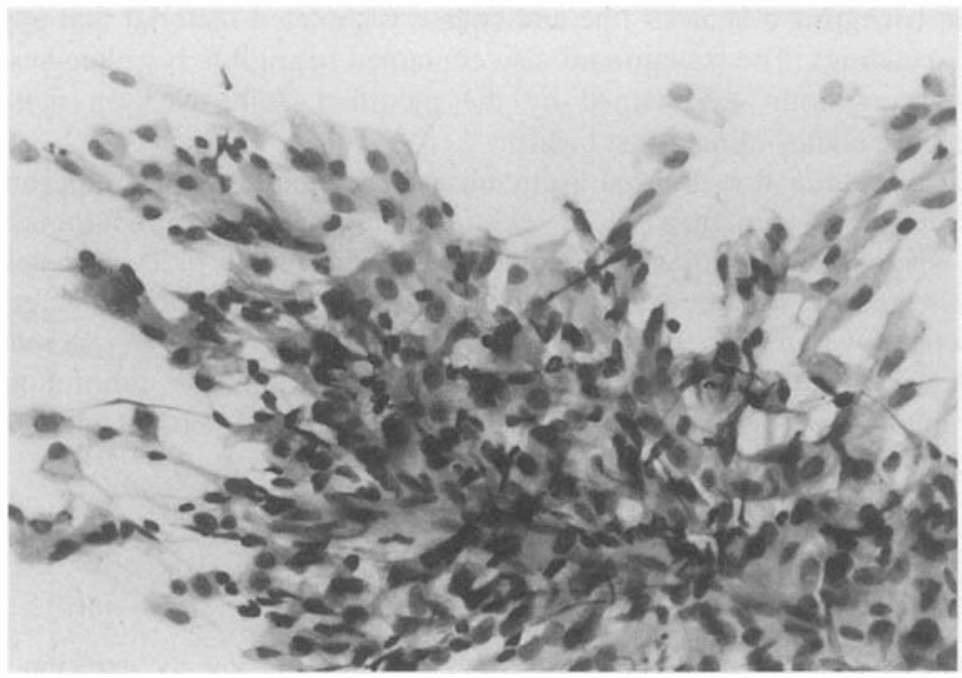

Figure 2. High power view of Figure 1. The spindle shaped cells are broader than those in paraffin sections. Note the endothelial core in the centre. (Papanicolaou $\times 200)$. 


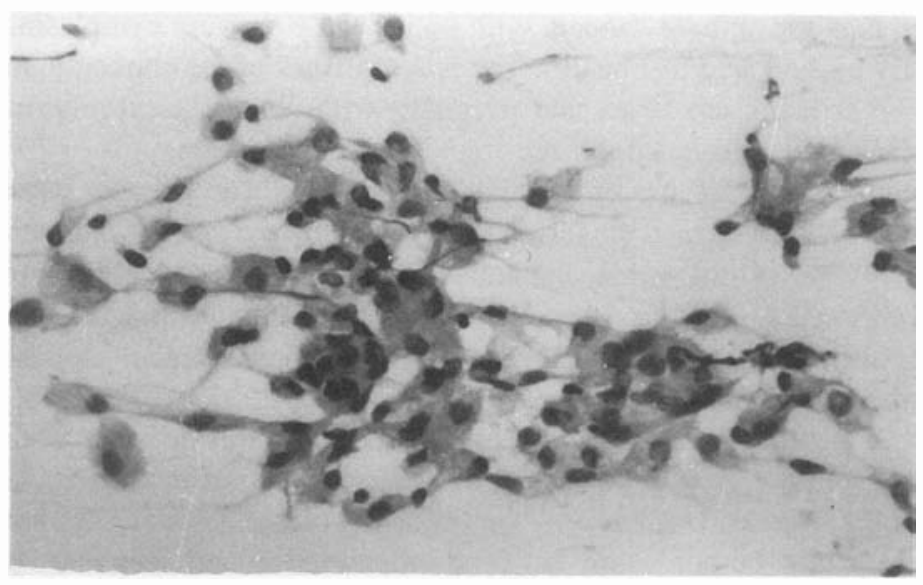

Figure 3. Cells from conventional lepromatous granuloma, irregular or polygonal with abundant cytoplasm. (Papanicolaou $\times 200)$.

seen in the smears reflects the architectural pattern of histoid leprosy. Tightly packed spindle shaped cells and forming bands are seen in the Papanicolaou-stained preparation. These cells appear little broader than those in histological sections, and the nuclei are also seen more prominently. An interesting finding in the smears is that the spindle-shaped histocytes are found around an endothelial vascular core (Figure 3), a feature not described in histological sections.

Histoid leprosy was first described by Wade ${ }^{5}$ in 1963 to distinguish a relatively unusual form of lepromatous leprosy with characteristic clinical and histological features. The cellular morphology has been subsequently confirmed by several workers. ${ }^{6-8}$ Essentially, the lesion is circumscribed, clearly separated by a pseudocapsule from the conventional granuloma of lepromatous leprosy. The cells of the histoid lesion are distinct from the cells of conventional lepromatous granuloma found just adjacent to it. The macrophages in the

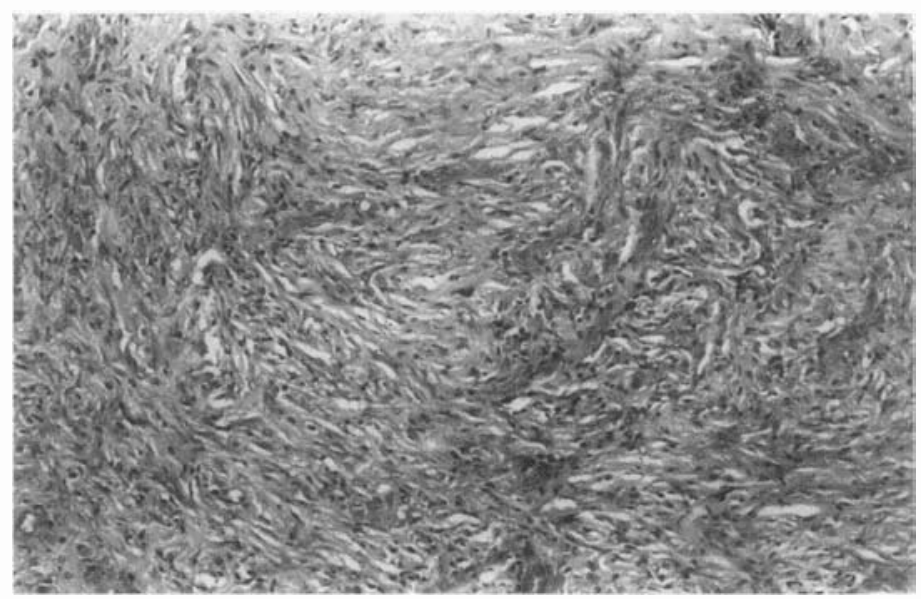

Figure 4. Paraffin section picture showing bands of thin spindle shaped cells forming whorls. $(\mathrm{H} \& \mathrm{E} \times 200)$. 
histoid type are thin and spindle-shaped, with poor evenly staining cytoplasm. They contain numerous tightly packed acid fast bacilli. The macrophages of the conventional lepromatous lesion, on the other hand, are large, and irregular, with abundant cytoplasm that is highly vacuolated. Acid fast bacilli in 'globi' are found in the cytoplasm.

In the present case, both types of cells have been described. The first pattern with bands of spindle-shaped cells confirmed the lesion to be of the histoid variety. The second pattern of loose or small groups of polygonal, vacuolated cells obviously comes from the adjacent lesion of conventional lepromatous leprosy. It has been pointed out that these cells were mostly found in the second aspiration. It seems likely that on repeated aspiration, the needle must have hit the conventional lepromatous granuloma.

The origin of histoid leprosy is not clear. Job et al. ${ }^{9}$ consider that histoid lesion is a result of alteration in the growth pattern of Mycobacterium leprae, possibly due to loss of immunity in localized areas. Desikan and lyer ${ }^{7}$ consider on the basis of morphological criteria that histoid lesions may be borderline between inflammation and neoplasm, but evidence is in favour of an inflammatory granuloma. Nevertheless, histoid leprosy presents interesting clinical and morphological features. It is therefore necessary to recognize and carefully follow up cases of histoid leprosy with regard to drug sensitivity, occurrence of reaction and relapse. Quite of ten, nodules of lepromatous leprosy are confused with histoid nodules. The distinction is only on cellular morphology. Cytodiagnosis by FNAC is therefore an easy tool to recognize the histoid variety of lepromatous leprosy. However, for management and follow up, skin smear examination would be a better indicator of bacterial load, since it is an accepted tool, needs less expertise and gives a better overall picture of changes in bacterial index.

\section{References}

${ }^{1}$ Gupta SR, Kumar B, Kaur S. Aspiration cytology of lymph nodes in leprosy. Int J Lepr, 1981; 49: 9-15.

2 Cavett JR, MacAtee R, Ramsy I. Hanson's disease (leprosy)—diagnosis by aspiration biopsy of lymph nodes Acta Cytol, 1966; 30: 189-193.

3 Singh H, Bhatia A, Arora VK, Bhattacharya SM. Fine needle aspiration cytology of lepromatous leprosy. Lepr Rev, 1998; 69: 145-150.

${ }^{4}$ Malik A, Bhatia A, Singh H et al. Fine needle aspiration cytology of reaction in leprosy. Acta Cytol, 1999; 43: 771-776.

5 Wade HW. The histoid variety of lepromatous leprosy. Int J Lepr, 1963; 31: 129-142.

${ }^{6}$ Mansfield R. Histoid leprosy. Arch Pathol, 1963; 87: 580-585.

7 Desikan KV, lyer CGS. Histoid variety of lepromatous leprosy - a histopathologic study. Int J Lepr, 1972; 40: 149-156.

8 Girdhar A, Lavania RK, Malaviya GH, Girdhar BK. Histoid lesion in nerve of lepromatous patient. Lepr Rev, 1990; 61: $237-241$.

9 Job CK, Chacko CJG, Taylor PH. Electron microscopic study of histoid leprosy with special reference to histogenesis. Lepr lnd, 1977; 49: 467-471. 